\title{
Strengthening the Potential of Magnetic Resonance Cholangiopancreatography (MRCP) by a Combination of High-Resolution Data Acquisition and Omni-directional Stereoscopic Viewing
}

\author{
Tetsuya Yamagishi ${ }^{1}$, Karl Heinz Höhne ${ }^{1}$, Taku Saito ${ }^{2}$, Kimihiko Abe ${ }^{2}$, \\ Jiro Ishida $^{3}$, Ryuko Nishimura ${ }^{3}$, and Tadashi Kudo ${ }^{3}$ \\ ${ }^{1}$ Institute of Mathematics and Computer Science in Medicine (IMDM), \\ University Hospital Hamburg-Eppendorf, \\ House S14, Martinistraße 52, 20251 Hamburg, Germany \\ \{yamagishi, hoehne\} @uke.uni-hamburg.de \\ http://www.uke.uni-hamburg.de/institute/imdm/idv \\ ${ }^{2}$ Department of Radiology, Tokyo Medical University Hospital, \\ Nishi-Shinjuku 6-7-1, Shinjuku-ku, 160-0023 Tokyo, Japan \\ ${ }^{3}$ Department of Radiology, Saiseikai-Kawaguchi Hospital, \\ Nishi-Kawaguchi 5-12-1, Kawaguchi-shi,332-8558 Saitama, Japan
}

\begin{abstract}
In this paper, we present a combination of high-resolution (HR) data from magnetic resonance cholangiopancreatography (MRCP) and omnidirectional viewing of stereoscopic maximum-intensity-projection. Since the presented method takes advantage of the property of magnetic resonancehydrography (MR-hydrography) as one of the most selective signal acquisitions, the segmentation process is very simple, and loads in data-processing is drastically reduced. The source data acquisition was performed with a slice thickness of $0.8 \mathrm{~mm}$. Images created by this method are precise and realistic enough to enhance the diagnostic capabilities of MRCP. The stereoscopic viewing and omnidirectional surveying can facilitate an accurate grasp of the three-dimensional extent of the entire pancreaticobiliary system, even in the regions which can not be assessed, due to signal-overlay of either neighboring organs or between pancreaticobiliary ducts. We are convinced that the presented post-processing is less time consumption and have a potential of optimizing HR-MRCP data sets.
\end{abstract}

\section{Introduction}

For numerous clinical tasks, for instance screening, diagnosis and surgical planning, it is essential to understand a complex and often malformed structure of the pancreaticobiliary system. Endoscopic retrograde cholangiopancreatography (ERCP) formerly held an unchallenged position as the only approach to get a precise delineation of the pancreaticobiliary ducts. ERCP, as an invasive modality, is accompanied by various 
risks, such as endoscopic technical failures [1], iatrogenic pancreatitis and adverse effect of contrast media as well as X-ray exposure.

In diagnostic imaging of the pancreaticobiliary discipline, we have seen an explosive development during the last decades. Although magnetic resonance cholangiopancreatography (MRCP) has become a standard tool and has come into the limelight as one of the non-invasive modalities to obtain information about the pancreaticobiliary system, a precise description of the morphological structure remains difficult by conventional scanning $[1,2]$. Because of false positive and/ or negative findings of MRCP [1, 2], ERCP is, even now, entrusted particularly as a modality for the final diagnosis.

The inferior image quality of MRCP also depends on its low spatial resolution [1] especially in the longitudinal body axis due to the source image data thickness up to 2$5 \mathrm{~mm}$ [1-4]. With the advent of modern magnetic resonance imaging (MRI) scanners, a thin-slice data acquisition integrating high-speed sequence acquisition and a breathtriggered technique [4] with a precision sensor has become available. Thus spatial resolution of MRI has drastically improved with a slice thickness below $1 \mathrm{~mm}$, which is called high-resolution (HR) data acquisition. Even abdominal section, HR images with less unexpected position discrepancies between slices or less motion artifacts have become possible in routine scanning.

An imaging technique of MRCP, or 'hydrography' as it is commonly called, is one of the most selective data acquisitions of MRI, which is following the principle of detecting the intensified degree of free-water MR-signals such as from the pancreaticobiliary system without contrast medium. Therefore, three-dimensional (3D) volume models derived from HR-MRCP get a potential of playing an increasing role not only for research analyses but also for clinical application. This paper is to show that the combination of HR data acquisition and omni-directional stereoscopic viewing of MIP provides a decisive increase of diagnostic value of MRCP.

\section{Methods}

\subsection{MR-Hydrography Data Acquisition}

Image data sets were obtained from five male healthy volunteers (40-53 years old) with fully informed consent. They had no disease history of the pancreaticobiliary system. The contraindication of iron particle, anti-cholinergic agents or secretin was excluded. A pre-medication protocol was performed, 10-15 minutes before scanning, by an oral administration of ferrite ammonium citrate $(0.2 \%$ water solution of $600 \mathrm{mg}$, for reducing T2-weighted signal from gastrointestinal tracts [1]), a 20mg of intravenous infusion of scopolamine butylbromide for avoiding motion-artifact due to increasing peristalsis, and a 50-unit of intravenous infusion of secretin for inducing pancreatic juice production for adequate visualization of the pancreatic duct system. Non-contrast MR images were obtained with a heavily T2-weighted fast-spin echo technique (TR/ 1639, TE/ 900) in coronal sections (total 250-270 slices) with a fatsignal-suppression technique by chemical-shift saturation). Each cross-sectional 
source image acquisition was performed with a slice thickness of $0.8 \mathrm{~mm}$ and with no gap between two adjacent slices. The image matrix was $256 \times 256$. A Philips Gyroscan Intera scanner (1.5T) was used.

\subsection{Processing}

Image data sets exported from the scanner were stored in CD-ROM as compressed DICOM format via the intra-hospital network. Then, all data sets were imported to the visualization system 'VOXEL-MAN', developed at the Institute of Mathematics and Computer Science in medicine (IMDM), Hamburg, Germany [5-8]. Interactive segmentation was performed for the purpose of defining three objects (the pancreaticobiliary structure and, the duodenum and the jejunum, for important landmarks [1] as adjoining alimentary tracts). The segmented alimentary tracts were removed for the purpose of avoiding an overlay with the pancreaticobiliary structure [5], in order to facilitate inspections. Due to remaining partial volume voxels [8] the duodenum and jejunum are still visible as like a transparent. The Red/ Green stereoscopic MIP rendering and sequential rotating movie projected from every-degree were created. The reconstructed images were evaluated at least on around two axes: the longitudinal body axis ( $\mathrm{Y}$ axis) and the lateral body axis ( $\mathrm{X}$ axis). All processing can be completed in the 'VOXEL-MAN' system. Animated sequences are displayed by commercial movie software (Quick Time Movie), which is also available by a slide-show mode of a commercial image viewer (ACDsee).

\subsection{Evaluation of Performance}

The created movies displayed on PC were compared to the conventional MIP on plain films by 3 radiologists and 2 board-certified endoscopists.

Image quality and ease of recognition of the following points were assessed:

1. the pancreaticobiliary junction

2. the cystic duct

3. the distal segment of the main pancreatic duct (MPD)

An assessment of usefulness for film-reading was made according the following score: 1: Excellent (persuasive), 2: Fair, 3. Good, 4. Poor in every aspect

Easiness of image computing and value for clinical application was also estimated.

\section{Results}

The entire morphological visualizations of the detailed contours of the pancreaticobiliary ducts can be substantially improved. Images created by this method are also realistic (Fig. 1-4), and by omni-directional observation, even in regions which can not be assessed due to signal-overlay with neighboring organs or between pancreaticobiliary ducts, accurate recognition of the ducts structure has been facilitated (Fig. 1-4). 
The actual scanning time is around 15 minutes, which depend on examinee respiration. The actual data-processing time is within one hour.

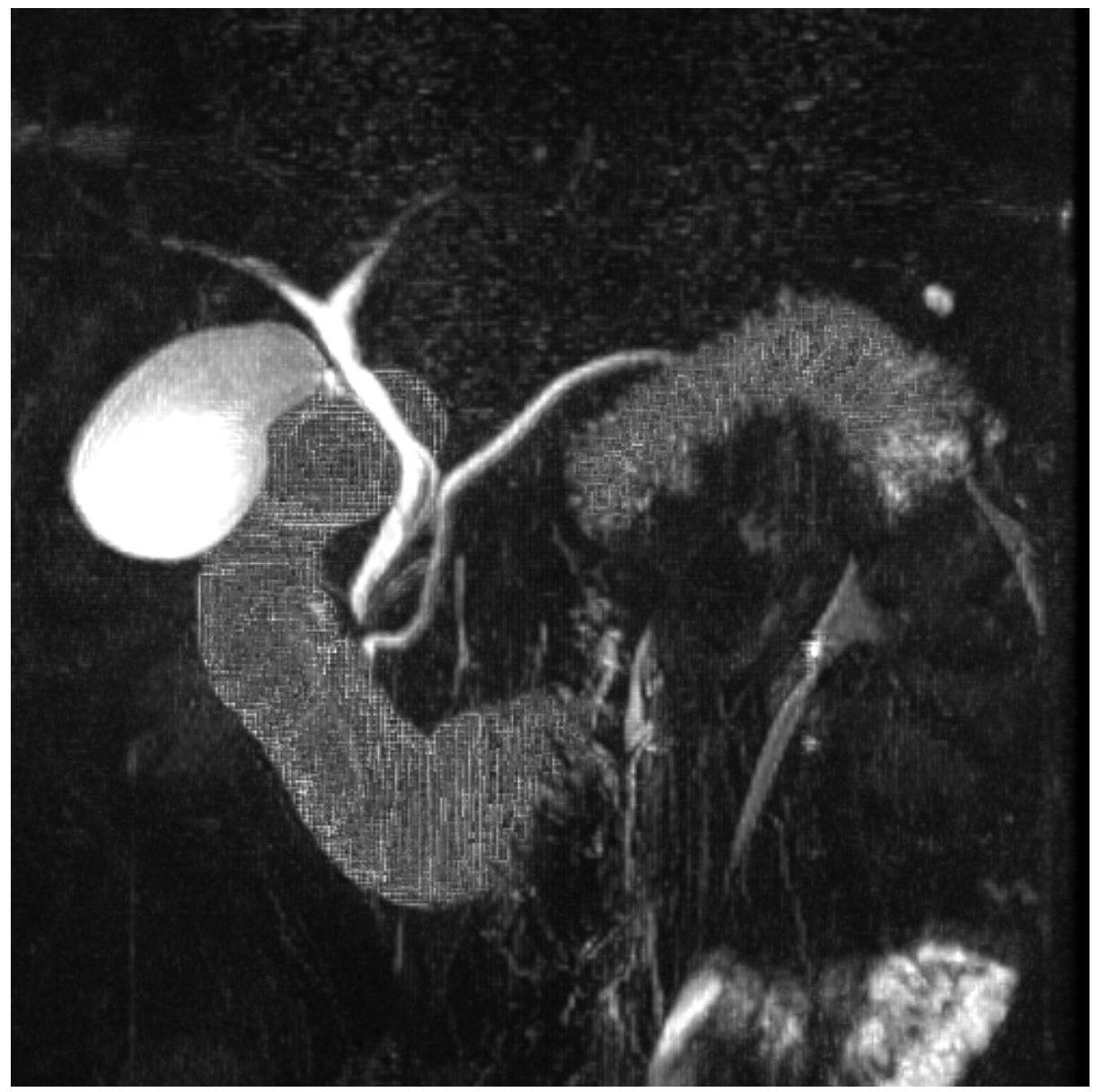

Fig. 1. The frontal view of a 40-year-old male. In this viewing direction, the different orifice of the common bile duct (CBD) and the MPD can easily be seen. But the distal segment of the MPD can hardly recognized. The small cystic lesion located in the tail of the pancreas can be suspected, but the precise location can not be identified

The very thin slice data acquisition enables a non-cleaved gapless MIPreconstruction even in a perpendicular projection compared to the original scanning direction of the source data acquisition, which is impossible under the condition of the conventional scanning with thick slices and gaps between slices (Fig.2). 


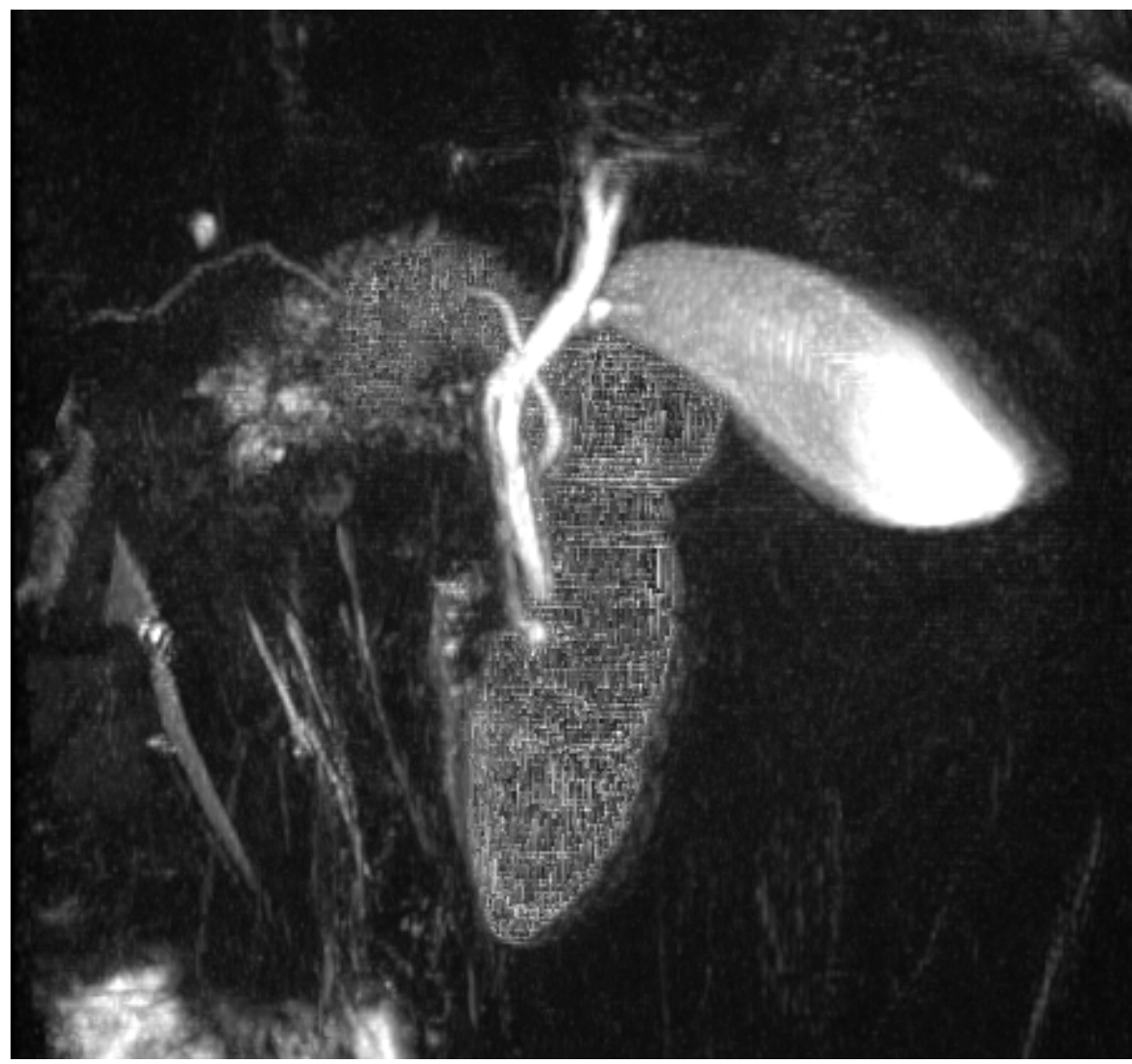

Fig. 2. The lateral view of the same case as shown in Fig. 1. By confronting with Fig. 1, we can firmly recognize the relative location between the distal segment of the MPD and the small cyst, which can not be seen in the frontal view by interference overlaid jejunum. The outlines of all objects are described without any gap or step edges because of the very thin slice of the source data acquisition

Panoramic viewpoints yield exact and continuous observation of the entire field of the pancreaticobiliary system (Fig.3). A small-degree rotation induces a benefit of intuitive understanding. (In a static MIP image we would not know which is in front or back). Furthermore, the advantages of visual continuity throughout the pancreaticobiliary system reduce the radiologists' burden of film reading as compared to the conventional single-shot images of MRCP.

All the radiologists and endoscopict participated in this study evaluated the created movies as excellent in the all aspects, except for one endoscopist evaluated fair in the recognition of the cystic duct in the case shown in Fig 3 and 4. 

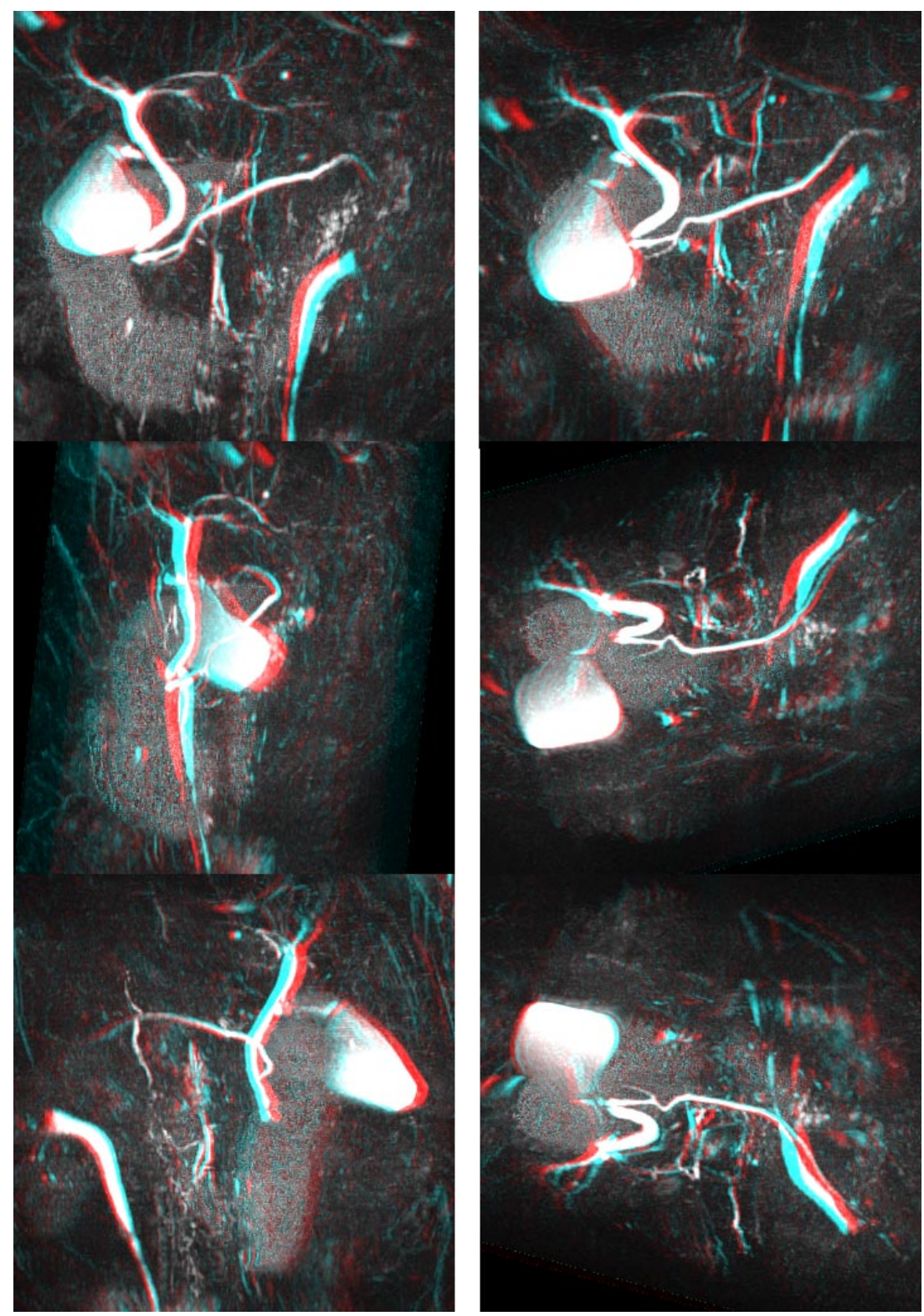

Fig. 3. The sequential stereoscopic view of a 46-year-old male. The left lane is around the vertical axis rotation and the right is transverse. The relative location of important landmarks of the pancreaticobiliary system can be recognized decisively, for example, the cystic duct and the CBD. In the stereoscopic view the perception is substantially improved

(These images must be viewed with RED/GREEN glasses). 


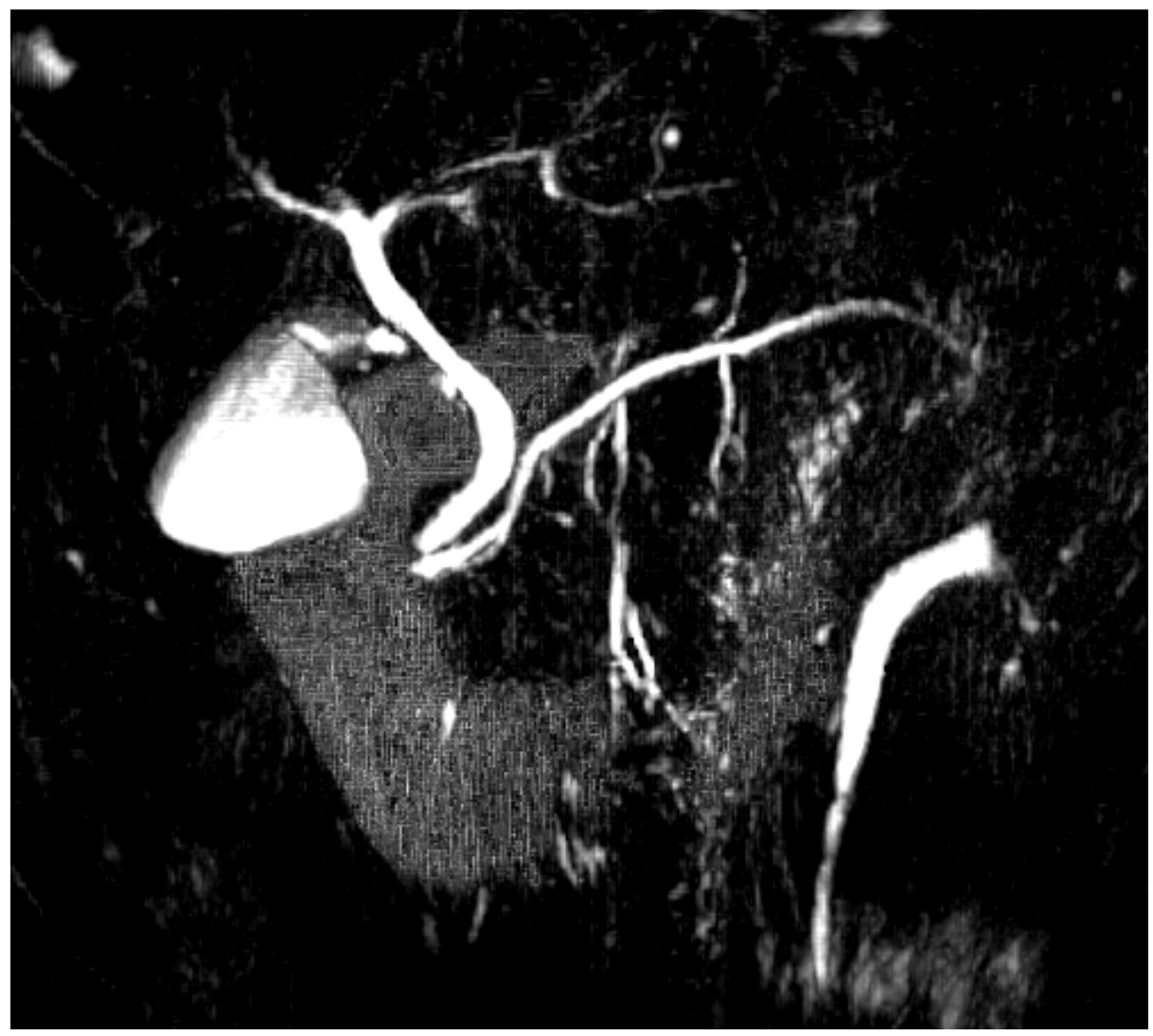

Fig. 4. The oblique view of the same case as shown in Fig. 3. We can easily pay attention to the separate channels of the pancreaticobiliary junction and the minor duodenal papilla is located distal as usual

\section{Discussion}

The quality of the final 3D images, in any rendering method, greatly depends on the excellence of the original data (the scanner strength; spatial resolution). In previous work concerning 3D MRCP, the source data slice thickness of $2-5 \mathrm{~mm}$ did not allow the rendering of precise and realistic images [1-4]. In spite of the image quality loss with decreasing slice thickness in MRI, the signal-to-noise ratio of hydrography is high enough to offer the potential of creating clear 3D images.

The HR data itself represents a large amount of images, so that if simply output on the plain films, film reading becomes a tedious task for diagnostic radiologists. We are convinced that the presented post-processing is an adequate tool for routine use of HRMRCP which is able to reduce the burden of film reading. 
We also want to point out that the speed of post-processing is very high, which has direct influence on availability for routine clinical use. Due to the high signal-to-noise ratio of the pancreaticobiliary system, that is to say the selectivity of the source data acquisition, we can expedite the segmentation, the first step of post-processing [5], because there is no necessity for the otherwise complicated procedure of defining target objects by specifying the lowest cut-off value of the gray scale. This dataprocessing requires neither complicated process nor a special tool. Ease of handling may contribute to cost-effectiveness and may boost the number of examinations with minimal manpower.

By omni-directional stereoscopic view, we are able to avoid false negative and /or positive findings due to overlaid high signal intensities. Improved image quality based on the HR data acquisition provides the possibilities for using in various clinical situations, such as the preoperative information and the early detection of neoplasm in the biliary and pancreatic region.

Since the thin-slice data acquisition requires long scanning time, an excellent breath-triggering system of the MR scanner is mandatory for HR data acquisition [4]. The developments of the modern scanners are making the solution of these problems.

We are convinced that the omni-directional stereoscopic HR-MRCP has the possibility of being considered as a useful option prior to ERCP and of reducing the cases of ERCP.

\section{References}

1. Arslan A. et al: Pancreaticobiliary diseases. Comparison of 2D single-shot turbo spin-echo MR cholangiopancreatography with endoscopic retrograde cholangiopancreatography. Acta Radiologica 41: 621-626, 2000

2. Watanabe Y. et al: High-resolution MR cholangiopancreatography. Crit Rev Diagn Imaging 39: 115-258, 1998

3. Holzknecht N. et al: Breath-hold MR cholangiography with snapshot techniques; prospective comparison with endoscopic retrograde cholangiography. Radiology 206: 657-664, 1998

4. Papanikolaou N. et al: Magnetic resonance cholangiopancreatography: comparison between respiratory-triggered turbo spin echo and breath hold single-shot turbo spin echo sequences. Magn-reson-imaging 17: 1255-1260, 1999

5. Schiemann T. et al: Interactive 3D-Segmentation: Visualization in Biomedical Computing 1808: 376-383, 1992

6. Hoehne KH. et al: A realistic model of human structure from the visible human data: Method Inform Med 40: 83-89, 2001

7. Pommert A. et al: Creating a high-resolution spatial/ symbolic model of the inner organs based on the Visible Human: Medical Image Analysis 5: 221-228, 2001

8. Hoehne KH. et al: 3D Visualization of tomographic volume data using the generalized voxel model: The Visual computer 6: 28-36, 1990 